\title{
Impressão de Protótipo Tridimensional (P3d) de Coração para Aplicação na Cardiologia Pediátrica: Uma Experiência Inicial
}

\author{
Three-Dimensional-Printed Heart Prototype for Application in Pediatric Cardiology: An Initial Experiment
}

\author{
Maíra Levorato Basso, ${ }^{1}$ Alessandra Möbius Gebran, ${ }^{2}$ Julia Dullius Oliveira, ${ }^{2}$ Katrin Möbius Gebran, ${ }^{2}$ Letícia \\ Carlota Bonatto, ${ }^{2}$ Maria Cecília Knoll Farah ${ }^{1}$ \\ Hospital Pequeno Príncipe, ${ }^{1}$ Curitiba, PR - Brasil \\ Faculdades Pequeno Principe, ${ }^{2}$ Curitiba, $P R-$ Brasil
}

\section{Introdução}

A tecnologia de impressão de protótipos tridimensionais (P3D) desenvolve-se rapidamente na medicina. Estudos recentes mostram a importância de sua aplicabilidade na cardiologia pediátrica.

Profissionais, clínicos e cirurgiões fazem uso de tecnologias bidimensionais como a ecocardiografia, a angiotomografia computadorizada (ATC) e a ressonância magnética para caracterizar estruturas e entender a complexidade das doenças. De modo geral, essas imagens são projetadas e estendidas em tela plana, de modo a não representarem o tamanho real das estruturas, a percepção de profundidade ou a proximidade entre elas. ${ }^{1}$

As cardiopatias congênitas têm prevalência de 9,1/1.000 nascidos vivos $^{2}$ e geram consequências hemodinâmicas e funcionais significativas, sendo responsáveis por $6 \%$ dos óbitos de crianças no primeiro ano de vida no Brasil. ${ }^{3}$ A diversidade e a complexidade das cardiopatias exigem do profissional a compreensão detalhada da anatomia no plano terapêutico, uma conduta adequada e a explicação didática aos familiares sobre a doença. ${ }^{4,5}$

Este estudo fez a impressão em P3D de cardiopatias congênitas a partir de imagens de arquivo de ATC e também como experiência inicial visando a construir uma maior evidência científica.

\section{Métodos}

Trata-se de um estudo descritivo e observacional no qual não foi aplicado um instrumento de comparação numérica. No entanto, visa a ressaltar uma experiência inicial, considerando a evidência presente na literatura médica sobre

\section{Palavras-chave}

Cardiopatias Congênitas; Angiografia Coronária/métodos; Tomografia Computadorizada por Raios X/métodos; Impressão Tridimensional/tendências.

\footnotetext{
Correspondência: Maíra Levorato Basso •

Hospital Pequeno Principe - Rua Desembargador Motta, 1070.

CEP 80250-060, Curitiba, PR - Brasil

E-mail: mairalevorato@gmail.com

Artigo recebido em 03/02/2020, revisado em 21/08/2020, aceito em 09/09/2020
}

DOI: https://doi.org/10.36660/abc.20200086 o uso da tecnologia 3D e os benefícios na compreensão das patologias cardíacas congênitas e sua importância em nosso meio. Imagens representativas das cardiopatias foram obtidas de arquivos de ATC, escolhidas dentre as que apresentavam maior complexidade anatômica e de acordo com a obtenção de melhores informações estruturais com a reprodução tridimensional. As cardiopatias selecionadas foram: atresia pulmonar com comunicação interventricular (APu+CIV) e colaterais sistêmico pulmonares, e hipoplasia do coração esquerdo (SHCE) após a colocação de stent no canal arterial e cerclagem dos ramos da artéria pulmonar.

As imagens foram obtidas através de tomógrafo GE Revolution com 512 detectores e modulação eletrocardiográfica, posteriormente tratadas no software Slic3r para segmentação estrutural. Para a confecção dos protótipos, foi utilizado um filamento termoplástico de ácido poliático com 0,2 mm de espessura e apoios em PVA no equipamento de impressão ZMorph VX/E. Após a impressão, os P3D foram comparados visualmente pelos autores com as imagens de ATC.

\section{Resultados}

Ao comparar visualmente as imagens da ATC com a impressão 3D, foi verificada a compatibilidade anatômica entre elas. Importante ressaltar que a percepção de detalhes importantes da anatomia no P3D foi evidenciada sob diversas perspectivas. No caso de APu + CIV, a compreensão da relação espacial entre as artérias colaterais sistêmico pulmonares originadas da aorta e os ramos pulmonares foi facilitada, bem como a observação comparativa das cavidades ventriculares (Figura 1). No caso de SHCE, foi visto o grau de hipoplasia da aorta ascendente e a presença de estenose do ramo pulmonar, além das relações espaciais entre elas (Figura 2). O tamanho dos modelos reconstituídos correspondeu à anatomia dos pacientes, possibilitando o estudo comparativo das dimensões entre as estruturas, o que pode colaborar na estratégia de tratamento cirúrgico. A possibilidade de ter o P3D em mãos e observar a anatomia sob diferentes ângulos tornou a compreensão das cardiopatias fácil e esclarecedora.

\section{Discussão}

A impressão 3D é uma tecnologia que busca a complementação dos exames convencionais, uma vez que torna possível compreender melhor a malformação cardíaca. Ela proporciona o estudo detalhado sobre a localização, o comprimento, a extensão e a relação entre as estruturas 

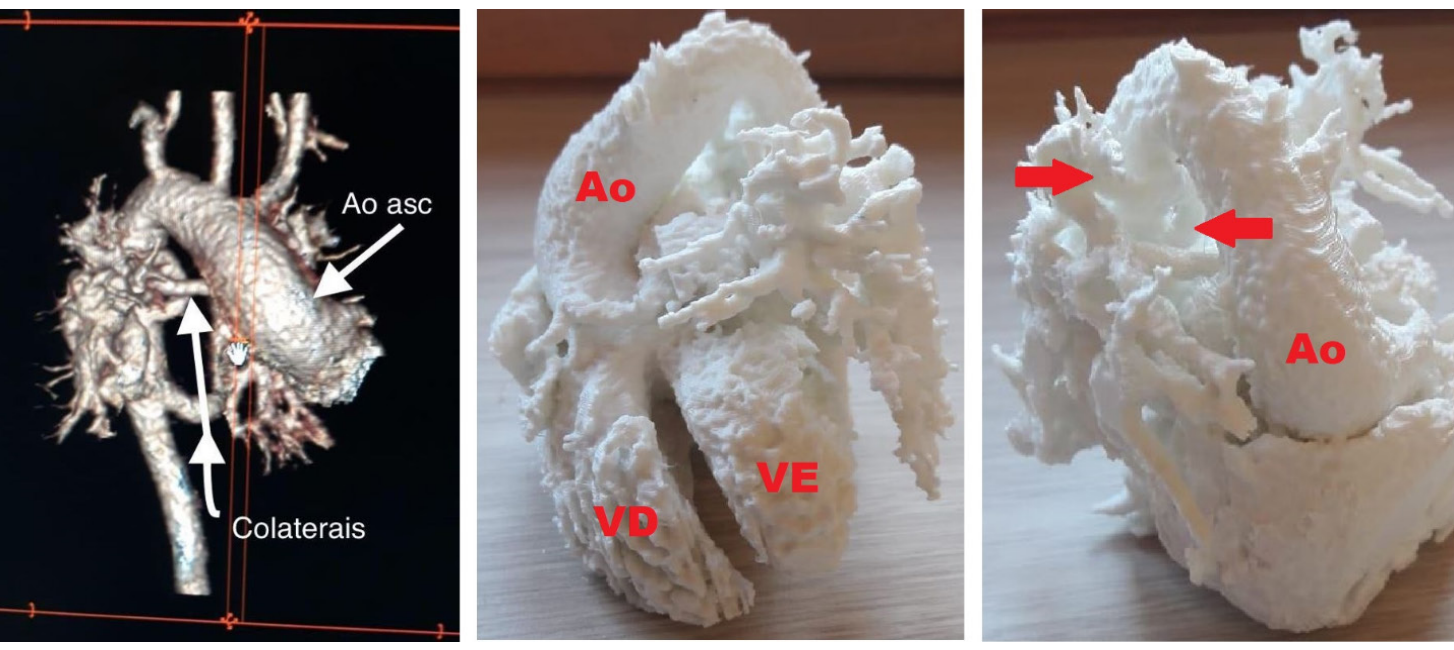

Figura 1 - Caso APu+CIV. À esquerda: ATC - setas indicam a aorta e a presença de artérias colaterais sistêmico pulmonares emergindo da aorta ascendente. À direita - P3D: visão frontal e lateral direita; Ao: aorta; VE: ventrículo esquerdo; VD: ventrículo direito.
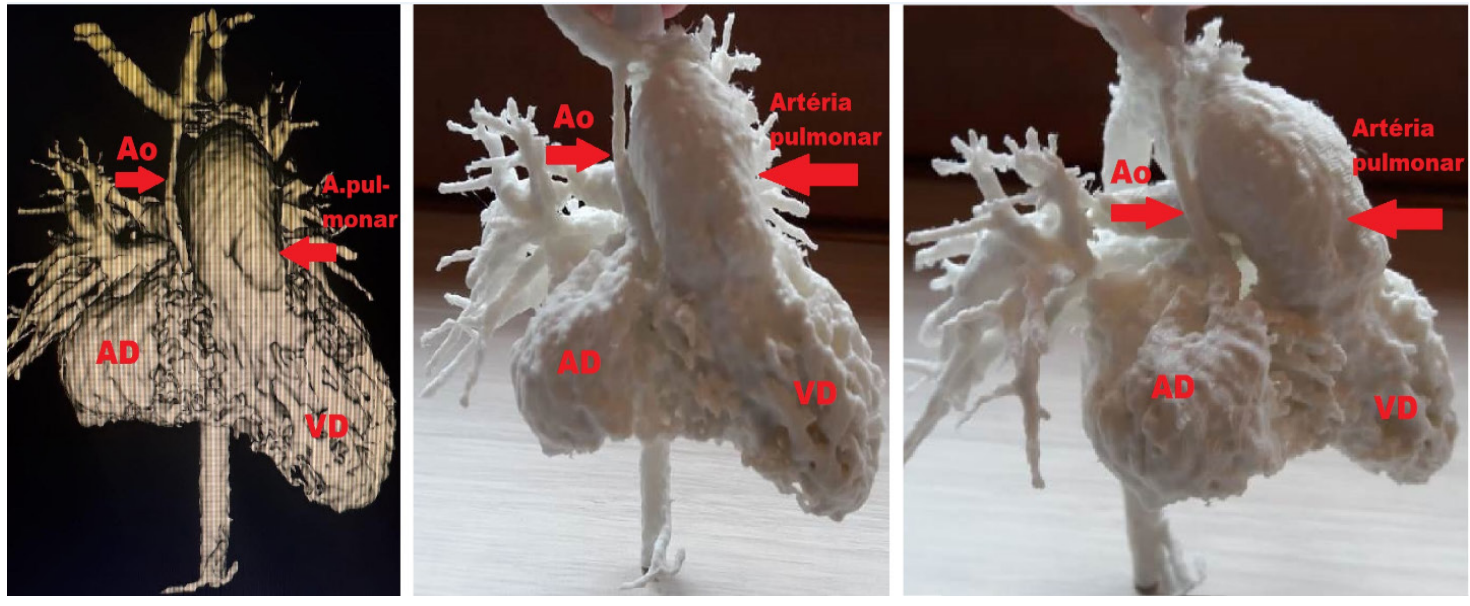

Figura 2 - Caso SHCE. À esquerda: ATC - setas indicam a aorta e a artéria pulmonar. À direita - P3D: visão lateral; Ao: aorta; AD: átrio direito; VD: ventrículo direito.

malformadas, o que auxilia no planejamento cirúrgico e na identificação de detalhes anatômicos de pacientes submetidos a intervenções prévias. ${ }^{6}$ Por conseguinte, beneficia profissionais na ampliação do conhecimento da cardiopatia e proporciona um maior grau de segurança na escolha de técnicas cirúrgicas. ${ }^{7}$ Estudos em pacientes com atresia pulmonar e defeito do septo ventricular mostraram que P3D possibilitou a visualização de $96 \%$ da maioria das artérias colaterais aortopulmonares, se comparada ao intraoperatório. Dessa forma, focaliza a intervenção por cateterismo e reduz o tempo de procedimento, de exposição à anestesia, de fluidoterapia e do uso de contraste em procedimentos hemodinâmicos. ${ }^{6-8}$

A tecnologia P3D também pode ser utilizada em simulações cirúrgicas, visando a detectar a necessidade de adaptações no planejamento cirúrgico, reduzir complicações, obter bons resultados nos pós-procedimentos e promover o treinamento de estudantes e médicos. ${ }^{1}$ Alguns protótipos apresentam alta flexibilidade e não exigem manuseio especial, podendo ser aplicados antes e durante a intervenção cirúrgica. ${ }^{9}$

Na educação médica, ela possibilita ao graduando e ao médico-residente um maior entendimento da patologia e orientação espacial das estruturas. Com as dificuldades crescentes na obtenção de cadáveres para estudo, o uso de P3D é uma importante opção no ensino sobre a anatomia humana em escolas médicas. ${ }^{10}$

No contexto da relação entre médico e paciente, a possibilidade de os pais segurarem o protótipo em suas próprias mãos e visualizar os detalhes anatômicos descritos pelo profissional de saúde proporciona um melhor entendimento tanto da fisiopatologia relacionada quanto 


\section{Carta Científica}

dos sintomas observados no paciente quanto ao tratamento. É descrito na literatura médica que os P3D auxiliam no estreitamento desta relação e são úteis na compreensão das informações e aumentam o conhecimento e o engajamento de paciente e familiares no que diz respeito à doença. ${ }^{11}$

A impressão em P3D é uma tecnologia em construção. Ela ainda apresenta limitações e desafios para garantir melhor qualidade ao produto. Entre elas, podemos destacar a precisão da montagem, a construção de modelos com mesmas propriedades mecânicas dos tecidos, o menor tempo de preparação e o custo econômico. A possibilidade de imprimir em cores facilitaria a identificação de diferentes tipos de estruturas como ventrículos, artéria pulmonar, ramos e aorta, principalmente no tocante ao ensino de graduação.

As cardiopatias representadas pelos protótipos satisfizeram as representações tridimensionais fidedignas às imagens obtidas nos exames de ATC, que foram utilizados como base para o estudo. A realização do P3D cardíaco é viável em nosso meio e pode ser uma ferramenta útil. Ela é capaz de auxiliar o clínico e a equipe cirúrgica na decisão terapêutica, o aprendizado do aluno no ensino médico de graduação, na especialização e na pós-graduação, o treinamento de habilidades cirúrgicas e o esclarecimento aos familiares da criança sobre a cardiopatia em tratamento.

Comentário: na publicação deste texto, devemos levar em consideração que as imagens dos $\mathrm{P} 3 \mathrm{D}$ perdem muito de seu impacto visual se comparadas com a observação direta e real da peça em mãos.

\section{Contribuição dos Autores}

Concepção e desenho da pesquisa e Revisão crítica do manuscrito quanto ao conteúdo intelectual importante: Basso ML, Farah MCK; Obtenção de dados, Análise e interpretação dos dados e Redação do manuscrito: Gebran AM, Oliveira JD, Gebran KM, Bonatto LC.

\section{Potencial conflito de interesses}

Declaro não haver conflito de interesses pertinentes.

\section{Fontes de financiamento}

O presente estudo não teve fontes de financiamento externas.

\section{Vinculação acadêmica}

Não há vinculação deste estudo a programas de pósgraduação.

\section{Aprovação ética e consentimento informado}

Este artigo não contém estudos com humanos ou animais realizados por nenhum dos autores.

\section{Referências}

1. Sundararaghavan S. Three-dimensional-printed cardiac prototypes in complex congenital cardiac defects: new technology with exciting possibilities. Ann Pediatr Card. 2017;10(2):114-6.

2. Sodian R, Stefan W, Markert M, Rassoulian D, Kaczmarek I, Lueth TC et al. Stereolithographic models for surgical planning in congenital heart surgery. Ann Thorac Surg. 2007;83:1854-7.

3. Lodziński P, Balsam P, Peller M, Gawałko M, Opolski G, Grabowski M. Threedimensional print facilitated ventricular tachycardia ablation in patient with corrected congenital heart disease. Cardiology J. 2017; 24(6):684-85.

4. van der Linde D, Konings EEM, Slager MA, Witsenburg M, Helbing WA, Takkenberg JJM et al. Birth prevalence of congenital heart disease worldwide. A systematic review and meta-analysis. J Am Coll Cardiol. 2011;58(21):2241-7.

5. Pinto Jr VC. Avaliação da política nacional de atenção cardiovascular de alta complexidade com foco na cirurgia cardiovascular pediátrica [dissertação]. Fortaleza: Universidade Federal do Ceará; 2010.

6. Valverde I. Impresión tridimensional de modelos cardiacos: aplicaciones en el campo de la educación médica, la cirugía cardiaca y el intervencionismo estructural. Rev Esp Cardiol. 2017; 70(4):282-91.
7. Kappanayil M, Koneti NR, Kannan RR, Kottayil B, Kumar K. Threedimensionalprinted cardiac prototypes aid surgical decisionmaking and preoperative planning in selected cases of complex congenital heart diseases: early experience and proof of concept in a resourcelimited environment. Ann Pediatr Card. 2017;10(2):117-125.

8. Ngan EM, Rebeyka IM, Ross DB, Hirji M, Wolfaardt JF, Seelaus R et al. The rapid prototyping of anatomic models in pulmonary atresia. J Thorac Cardiovasc Surg. 2006; 132(2):264-269.

9. Abudayyeh I, Gordon B, Ansari MM, Jutzy K, Stoletniy L, Hilliard A. A practical guide to cardiovascular $3 \mathrm{~d}$ printing in clinical practice: overview and examples. J Interv Cardiol. 2018;31:375-383.

10. Luo H, Meyer-Szary J, Wang Z, Sabiniewicz R, Liu Y. Three-dimensional printing in cardiology: Current applications and future challenges. Cardiol J. $2017 ; 24(4): 436-44$.

11. Biglino G, Koniordou D, Gasparini M, Capelli C, Leaver LK, Khambadkone S et al. Piloting the use of patient-specific cardiac models as a novel tool to facilitate communication during cinical consultations. pediatr cardiol. 2017; 38(4):813-8. 